\title{
Machine Learning Approach to Assess the Performance of Patch Based Leads in the Detection of Ischaemic Electrocardiogram Changes
}

\author{
Michael R Jennings ${ }^{1}$, Pardis Biglarbeigi ${ }^{1}$, Raymond R Bond ${ }^{1}$, Rob Brisk ${ }^{1}$, Daniel Güldenring ${ }^{2}$, Alan \\ Kennedy $^{1}$, James McLaughlin ${ }^{1}$, Dewar D Finlay ${ }^{1}$ \\ ${ }^{1}$ Ulster University, Newtownabbey, UK \\ ${ }^{2}$ HTW Berlin, Berlin, Germany
}

\begin{abstract}
Background: We have previously reported on the potential of patch-based ECG leads to observe changes typical during ischaemia. In this study we aim to assess the utility of patch-based leads in the detection of these changes. Method: Body surface potential maps (BSPM) from subjects $(n=45)$ undergoing elective percutaneous coronary angioplasty (PTCA) were used. The short spaced lead (SSL), that was previously identified as having the greatest ST-segment change between baseline and peak balloon inflation (PBI), was selected as the basis for a patch based lead system. A feature set of J-point amplitudes for all bipolar leads available within the same $100 \mathrm{~mm}$ region were included (n=6). Current 12-lead ECG criteria were applied to 12-lead ECGs for the same subjects to benchmark performance.

Results: The previously identified single SSL achieved sensitivity and specificity of $87 \%$ and $71 \%$ respectively using a Naive Bayes classifier. Adding other combinations of leads to this did not improve performance signifcantly. The 12-lead ECG performance was 62/93\% (sensitivity/specificity).

Conclusion: This study suggests that short spaced leads can be sensitive to ischaemic ECG changes. However, due to the short distance between leads, they lack the specificity of the 12-lead ECG.
\end{abstract}

\section{Introduction}

The 12-lead ECG remains the most common tool in the detection of ST-elevation myocardial infarction (STEMI) [1]. It has high specificity in STEMI detection, however, it is often insensitive [2]. Additionally, the placement of electrodes across the torso is inconvenient compared to other systems designed for longer term monitoring that use fewer electrodes. Machine learning (ML) might be an effective method for detecting STEMI when trained using ECG data, particularly when applied to pre-hospital ad- mission [3]. ML has allowed more diverse methods of classifying cardiac abnormalities than the 12-lead ECG [4]. STEMI can occur in different coronary arteries of the heart. Vessel-specific leads (VSL) have been introduced, however, these may be inconvenient when used in a clinical setting [5]. Furthermore, reduced-lead systems such as smartphone-based devices are limited by systematic error between electrodes [6]. A practical and more convenient recording system with high sensitivity in the detection of STEMI has the potential to reduce door-to-balloon time [7]. Patch-based lead systems have been introduced for cardiac arrhythmia monitoring however there has been less emphasis on the development and reporting of systems designed for ischaemic heart disease [8]. Such devices are prone to placement errors, however, ML can detect misplacement [9]. We have previously introduced an SSL based on the greatest ST-segment changes across patients with ischaemic-type ECG changes [10]. The aim of this study is to assess the performance of an SSL-based system using machine learning in the classification of STEMI.

\section{Method}

\subsection{Data}

Data were described by Horacek et al. [5]. Data were collected from patients $(n=45)$ undergoing PTCA. Two ECG recordings were taken from each patient, one during rest and one during peak balloon inflation (PBI). A total of 90 recordings are available. Inflations were carried out in one of three coronary arteries: LAD $(n=15)$, LCX $(n=15)$ and RCA $(n=15)$. Each recorded lead were averaged to provide one cardiac cycle. Recordings were taken from 120-lead BSPMs at a sample rate of $500 \mathrm{~Hz}$. Three of the leads were distal limb leads of the 12-lead ECG. The BSPMs were expanded to the 352-node Dalhousie torso [11] using Laplacian interpolation. The fiducial points (QRS onset, J-point and end of the T-wave) were determined by an automated algorithm and reviewed manually. 


\subsection{Feature Extraction}

Our lead system was based on the previously introduced SSL sensitive to ST-segment changes [10]. In that work, a single bipolar lead with electrodes spaced $100 \mathrm{~mm}$ apart was proposed. This lead was selected based on the fact that exhibited the greatest J-point amplitude change between baseline and PBI across all subjects studied. The 100mm distance was chosen to be compatible with the amount of space that would typically be occupied by a single self contained patch. In the current work we have explored adding additional leads to the previously chosen SSL. Electrodes were positioned on the anterior torso. Specifically, between a region in the left precordium and a more inferior abdominal region. To increase spatial resolution, all possible bipolar leads within $100 \mathrm{~mm}$ of the SSL were added. A total of six bipolar leads were used: ST-sensitive $\left(S S L_{S T}\right)$, spatially orthogonal $\left(S S L_{\text {orth }}\right)$ and four complementary $\left(S S L_{3-6}\right)$. The purpose of this was to develop the basis for an ambulatory patch lead system.

The amplitude at the J-point was extracted as the feature for each SSL based on its importance in standard STEMI criteria [1]. This resulted in six features for each recording, one for each SSL. Recordings at rest were annotated as healthy (false), whereas PBI were annotated as STEMI (true).

\subsection{STEMI Detection}

Given that no criteria exist for the new leads, we employed a machine learning based approach to assess the performance of the extracted J-points at distinguishing between STEMI and ECGs recorded at rest. We also used the standard STEMI detection criteria, as applied to the standard 12-lead ECG that were extracted for the same patient. 12-lead ECG channels were extracted from each recording at both rest and PBI. Currently accepted STEMI criteria were employed based on J-point amplitudes, age and sex [1]. The criteria used did not include new Q-wave, STslope or T-wave changes. This was performed in MATLAB R2020a.

Three classifiers were used to detect STEMI. The C4.5 (J48) decision tree [12], multi-layer perceptron (MLP) and Naive Bayes (NB)[13] classifiers. These were performed using the WEKA 3.8.4 software. Three different combinations of SSLs were used for each classifier. The first involved all SSLs $(n=6)$. The second omitted the four complementary SSLs, leaving only the $S S L_{S T}$ and $S S L_{\text {orth }}$ leads. The third involved only $S S L_{S T}$. 10-fold cross validation was used to validate classifier performance.
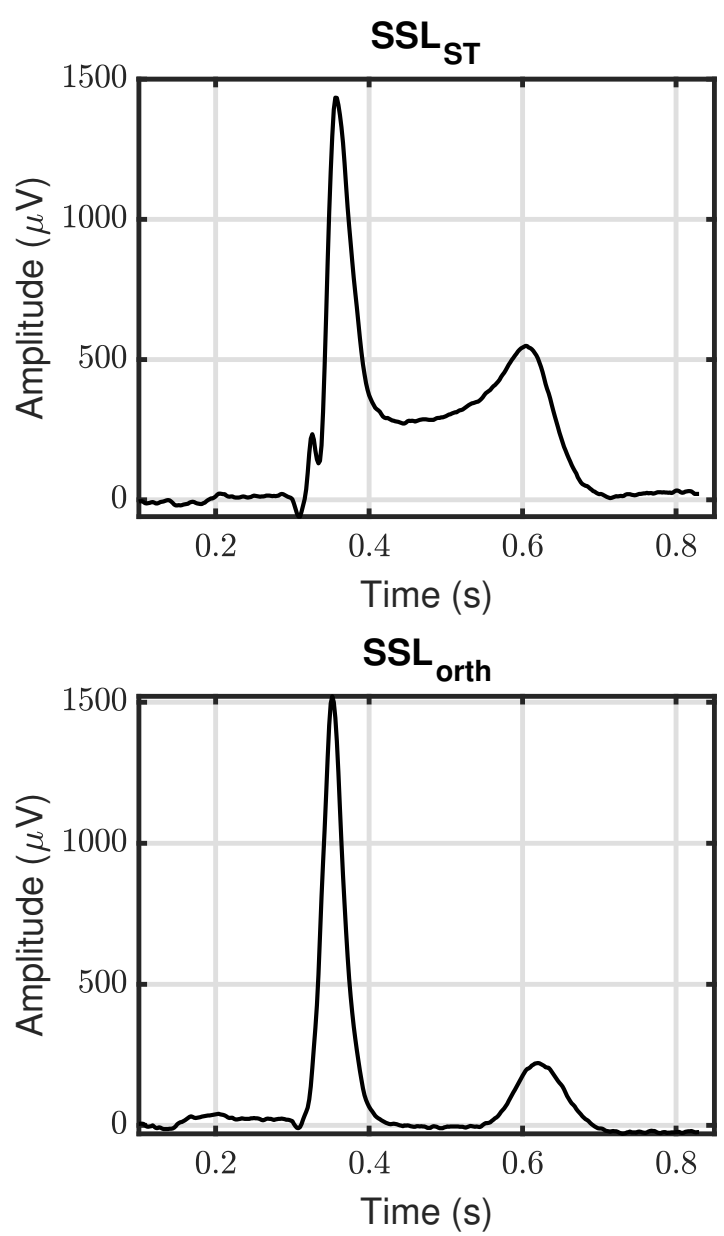

Figure 1. $S S L_{S T}$ and $S S L_{\text {orth }}$ plotted for one recording. PBI during RCA inflation

\section{Results}

The sensitivity (Se), specificity ( $\mathrm{Sp}$ ) and F1 score (F1) were calculated for each SSL combination and classifier. Table 1 shows the results. The highest overall performance was using only $S S L_{S T}$ with a Naive Bayes algorithm. The sensitivity and specificity were $86.7 \%$ and $71.1 \%$ respectively.

The chosen classifiers generally exhibited greater sensitivity than specificity in their default configurations. It should be noted that these classifiers do not offer the facility to easily adjust thresholds towards either sensitivity or specificity. Further work is required to facilitate this or a ROC curve based approach. The C4.5 and Naive Bayes classifiers had similar performances across lead combinations. Overall, sensitivity and specificity were higher when only $S S L_{S T}$ was used. The MLP is an exception to this. Performance was considerably reduced when a single SSL was used with MLP. This classifier is a feed-forward classifier that relies on back-propagation. A lack of input fea- 
Table 1. Classifier performance for each combination of SSLs

\begin{tabular}{ccccc}
\hline & & \multicolumn{3}{c}{ Short Spaced Lead (SSL) Combination } \\
Classifier & & $S S L_{S T}$ & $S S L_{S T} \& S S L_{\text {orth }}$ & All SSLs \\
\hline \multirow{3}{*}{ C4.5 (J48) } & Se (\%) & 86.7 & 86.7 & 80.0 \\
& Sp (\%) & 68.9 & 66.7 & 60 \\
& F1 (\%) & 79.6 & 78.8 & 72.7 \\
MLP & Se (\%) & 53.3 & 80.0 & 75.6 \\
& Sp (\%) & 46.7 & 68.9 & 68.9 \\
& F1 (\%) & 51.6 & 75.8 & 73.1 \\
Naive Bayes & Se (\%) & 86.7 & 84.4 & 82.2 \\
& Sp (\%) & 71.1 & 66.7 & 66.7 \\
& F1 (\%) & 80.4 & 77.6 & 76.3 \\
\hline
\end{tabular}

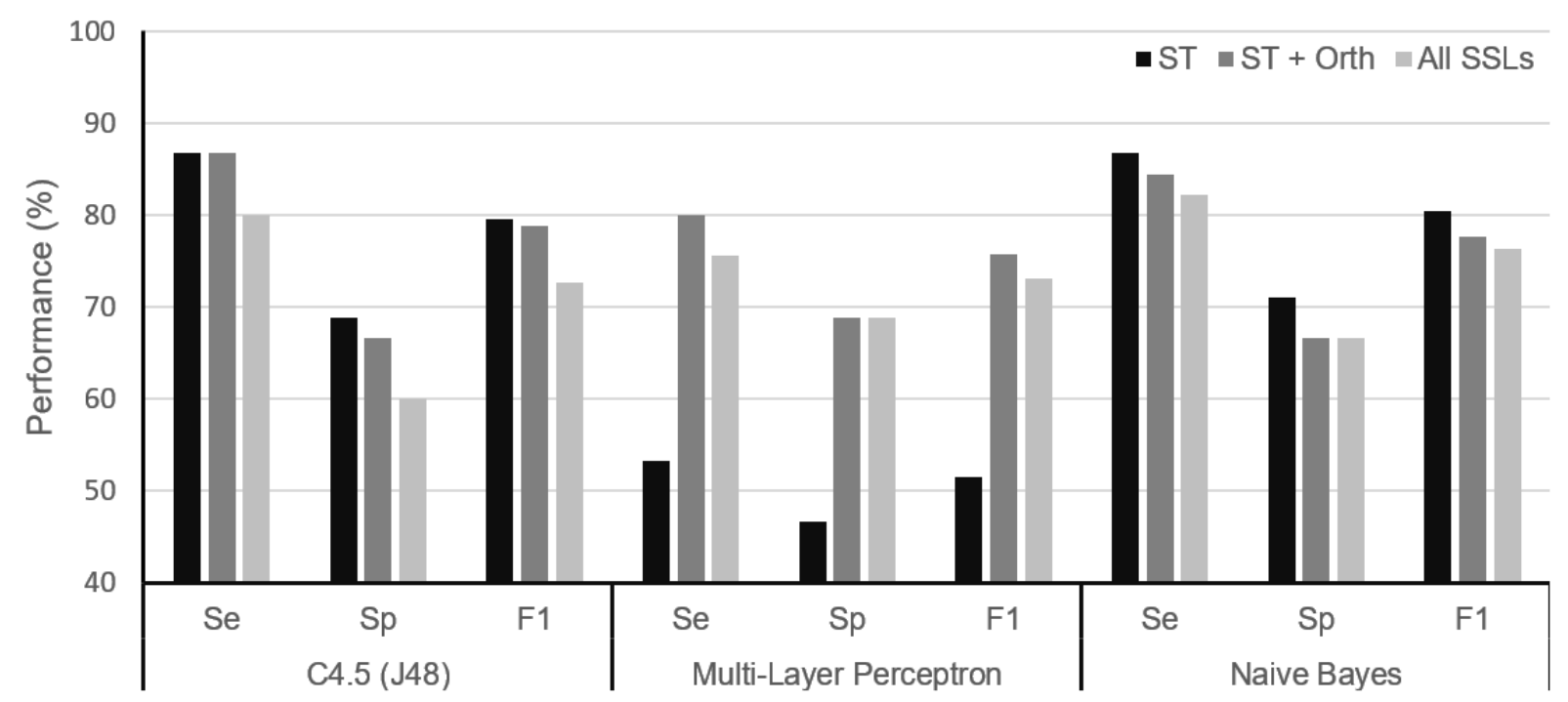

Figure 2. Sensitivity (Se), specificity (Sp) and F1 score (F1) for different combinations of short-spaced leads (SSL)

tures may negatively affect its performance [14].

The 12-lead ECG classifier based on current diagnostic criteria had a sensitivity and specificity of $62 \%$ and $93 \%$, respectively. This is comparable to the known 12-lead diagnostic capability [2]. Unlike the SSL-based classifiers, the specificity is higher than sensitivity. The 12-lead ECG has a higher spatial resolution than an SSL patch which may increase specificity to ischaemic-type ECG changes.

Compared to the 12-lead ECG, the $S S L_{S T}$-based Naive Bayes classifier was more sensitive. The bipolar lead of the SSL is across the highest amplitude gradient on the torso during the ST-segment. This will emphasise J-point changes in this lead during ischaemia. The SSL is unspecific in comparison. There is a lower distance between electrodes compared to the 12-lead ECG. The 12-lead criteria used age, sex and J-point changes, however, the SSLs only used J-point changes.
Electrode placement errors affect the diagnostic capability of lead systems. An SSL patch may be affected more than the 12-lead ECG by misplacement. This may reduce the sensitivity of STEMI detection.

\subsection{Utility}

An SSL patch is more convenient than the 12-lead ECG. An unskilled operator could fit the patch prior to paramedical intervention and recording of the 12-lead ECG. Additionally, such a device could be complementary to the 12lead ECG to increase performance. One proposed design for such a device is using a four-electrode, two-lead patch. $S S L_{\text {orth }}$ can be used for QRS detection while $S S L_{S T}$ is used for ST-segment monitoring. Figure 1 shows two SSLs during PBI in the right-coronary artery.

There is no doubt that the performance of the 12-lead ECG will be superior to that with a greatly reduced num- 
ber of leads. Nevertheless, our work has indicated that there is potential to greatly reduce the recording complexity of the 12 lead ECG towards a patch based system. This may greatly streamline the acquisition process. Our work has introduced machine learning techniques and further refinement of these methods could bring the performance of the patch based system closer to that of the 12-lead system. Further work is required to allow us to tune the ML techniques so that a better comparison can be made, in terms of the sensitivity and specificity balance, with the 12lead ECG. A larger dataset of ischaemic-type ECGs will also strengthen this comparison. Specifically we believe there is a need to further evaluate the proposed patch based leads in more complex MI disease cohorts, e.g. those with multi-vessel disease. In addition we envisage that our future work will also investigate the variations in placement that may be encountered in the use of such a patch based system whose application is not based on the well known anatomical landmarks associated with 12-lead precordial lead placement.

\section{Conclusion}

We have used ML techniques to evaluate the use of a patch-based SSL system in the detection of myocardial ischaemia. Out of nine different combinations of leads and classifiers, a single SSL coupled with a Naive Bayes classifier yielded the highest sensitivity/specificity combination $(86.7 \% / 71.1 \%)$. 12-lead ECG recordings and current diagnostic criteria were used for comparison purposes $(62 \% / 93 \%)$. Further research into patch placement, feature extraction and classification methods must be carried out to truly evaluate the lead system.

\section{Acknowledgements}

This project is part of the Eastern Corridor Medical Engineering centre (ECME). It is supported by the European Union's INTERREG VA Programme, managed by the Special EU Programmes Body (SEUPB).

\section{References}

[1] Thygesen K, Alpert JS, Jaffe AS, Chaitman BR, Bax JJ, Morrow DA, White HD. Fourth universal definition of myocardial infarction. European Heart Journal 2018; 24(3):107-138. ISSN 26187620.

[2] Perron A, Lim T, Pahlm-Webb U, Wagner GS, Pahlm O. Maximal increase in sensitivity with minimal loss of specificity for diagnosis of acute coronary occlusion achieved by sequentially adding leads from the 24-lead electrocardiogram to the orderly sequenced 12-lead electrocardiogram. Journal of Electrocardiology 2007;40(6):463-469. ISSN 00220736.

[3] Al-Zaiti S, Besomi L, Bouzid Z, Faramand Z, Frisch S,
Martin-Gill C, Gregg R, Saba S, Callaway C, Sejdić E. Machine learning-based prediction of acute coronary syndrome using only the pre-hospital 12-lead electrocardiogram. Nature Communications 2020;11.

[4] Islam Chowdhuryy MH, Sultana M, Ghosh R, Ahamed JU, Mahmood M. AI Assisted Portable ECG for Fast and Patient Specific Diagnosis. In International Conference on Computer, Communication, Chemical, Material and Electronic Engineering, IC4ME2 2018. Institute of Electrical and Electronics Engineers Inc. ISBN 9781538647752, 2018; 1-4.

[5] Horáček BM, Mirmoghisi M, Warren JW, Wagner GS, Wang JJ. Detection of myocardial ischemia by vesselspecific leads derived from the 12-lead electrocardiogram and its subsets. Journal of Electrocardiology 2008; 41(6):508-517. ISSN 0022-0736.

[6] Guldenring D, Finlay DD, Bond RR, Kennedy A, McLaughlin J. Limitations of the smartphone based single ECG-lead evaluation of STEMI. Journal of Electrocardiology 2018;51(1):e3. ISSN 00220736.

[7] Brown JP, Mahmud E, Dunford JV, Ben-Yehuda O. Effect of Prehospital 12-Lead Electrocardiogram on Activation of the Cardiac Catheterization Laboratory and Doorto-Balloon Time in ST-Segment Elevation Acute Myocardial Infarction. American Journal of Cardiology 2008; 101(2):158-161. ISSN 00029149.

[8] Guzik P, Malik M. ECG by mobile technologies. Journal of Electrocardiology 2016;49(6):894-901. ISSN 15328430.

[9] Rjoob K, Bond R, Finlay D, McGilligan V, Leslie SJ, Iftikhar A, Guldenring D, Rababah A, Knoery C, McShane A, Peace A. Data driven feature selection and machine learning to detect misplaced V1 and V2 chest electrodes when recording the 12-lead electrocardiogram. Journal of Electrocardiology 2019;57:39-43. ISSN 15328430.

[10] Jennings M, Guldenring D, Bond R, Rababah A, McLaughlin J, Finlay DD. ST Changes Observed in Short Spaced Bipolar Leads Suitable for Patch Based Monitoring. In Computing in Cardiology, volume 2019-Septe. IEEE Computer Society. ISSN 2325887X, 2019; 1-4.

[11] Horacek BM. Numerical model of an inhomogeneous human torso. Advanced Cardiology 1974;10(51).

[12] Quinlan, J R. C4.5: Programs for Machine Learning. San Mateo, California: Morgan Kaufmann Publishers, 2014. ISBN 1-55860-238-0.

[13] John GH, Langley P. Estimating continuous distributions in bayesian classifiers. In Eleventh Conference on Uncertainty in Artificial Intelligence. San Mateo: Morgan Kaufmann, 1995; 338-345.

[14] Elliott S. Active Control of Nonlinear Systems. In Signal Processing for Active Control. Elsevier, 2001; 367-409.

Address for correspondence:

Michael Jennings

NIBEC, Ulster University

NEWTOWNABBEY, United Kingdom

jennings-m5@ulster.ac.uk 